\title{
Radial Force Control of Multi-Sector Permanent Magnet Machines Considering Radial Rotor Displacement
}

\author{
G. Valente, L. Papini, A. Formentini, C. Gerada, P. Zanchetta
}

\begin{abstract}
A mathematical model enabling to predict the electromagnetic $x-y$ forces and torque for a given input current in a Multi-Sector Permanent Magnet Synchronous (MSPMS) machine is presented. The rotor static eccentricity is also accounted and the analytical calculations are validated by means Finite Element Analysis (FEA).

Furthermore, a novel force and torque control is proposed based on input current minimization and is applied to suppress the Unbalanced Magnetic Pull (UMP) caused by the rotor eccentricity. The effective operation of the force suppression technique is verified by means of FEA.
\end{abstract}

Index Terms-Radial force control, static eccentricity, stiffness matrix.

\section{INTRODUCTION}

I $\mathrm{n}$ recent years, permanent magnet synchronous machines (PMSMs) have received a growing attention in industrial applications such as automotive and aerospace [1] but find market also in process applications such as spindles, turbocharges, waste heat recovery systems and in pumping systems [2]. The study of the eccentricity in such machines is particularly interesting because it is one of the causes of acoustic noise and undesired vibrations [3]. The static eccentricity is one of the typical case study which are of interest when comes to investigate the rotor-dynamic of rotating components in electrical machines. A not perfect rotor centering can be caused, during the manufacturing process, by not accurate rotor balancing, bearing tolerances and by a misalignment introduced in the assembly process. The rotor static eccentricity can result in a reduced lifetime of the machine due to bearings wear and additional mechanical stresses on the rotating components.

Various approaches have been proposed to predict and study the effect of rotor eccentricity in PMSM. The analytical estimation of the flux density in the airgap is a well-known technique adopted to predict the electromagnetic forces in an electric machine. Several papers dealing with flux density prediction in PMSM can be found in the literature [4], [5]. In [6], [7] and [8], [9] an approximated method is introduced to calculate the flux density for rotor eccentricity and the resultant unbalanced radial force for synchronous reluctance and PMS motors, respectively. Furthermore, [10] presents a very detailed method to analytically calculate UMP exploiting the Maxwell stress tensor method.

In [11] a mathematical model of the torque-force characteristic was presented with the hypothesis of centered rotor. It led to an efficient radial force-torque control method for the MSPMS machine considered.

In the present work, the mathematical model proposed in [11] is elaborated to take the static rotor eccentricity into account. Furthermore, a detailed analytical calculation of the coefficients of the mathematical model will be studied.

In particular, the model relies on the airgap flux density prediction carried out in [4], [5], [12] for no eccentricity condition and [8], [9] for rotor eccentricity levering on the Maxwell stress tensors computation [10]. The analytical calculation is benchmarked against FEA. The mathematical model can be exploited to define a new radial force and torque control technique that allows to compensate undesired forces and is demonstrated on damping the UMP caused by the rotor static eccentricity.

\section{MACHINE CHARACTERIZATION}

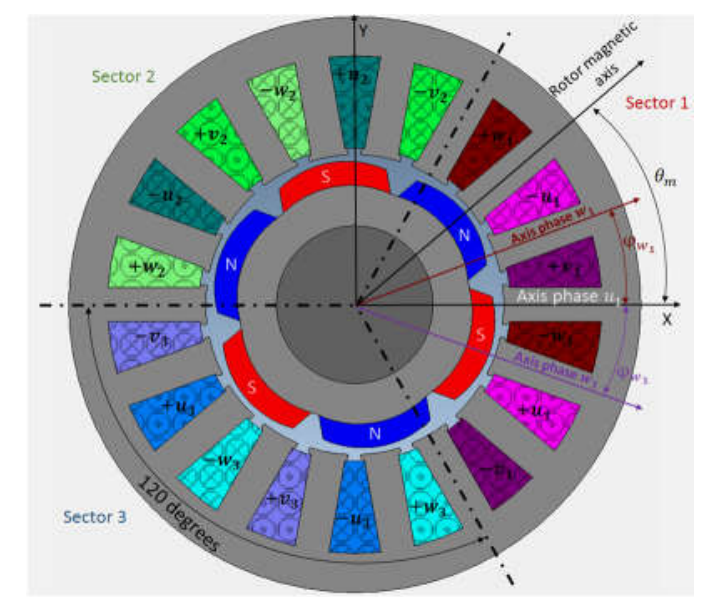

Fig. 1. -Cross-section of the 18 slots -6 poles -3 sectors PMSM considered.

\section{A. Machine Structure}

A multi-sector three-phase MSPMS machine is considered in this work, consisting of a conventional 18 slot -6 poles surface mounted PMSM but with a re-arranged winding configuration [11]. The distributed winding featuring a single slot/pole/phase are highlighted in Fig. 1 together with the cross sectional geometrical structure of the MSPMS machine considered. The $n_{s}=3$ machine sectors are selected to dedicate a single winding set to each pole pair as highlighted

G. Valente, L. Papini, A. Formentini, C. Gerada, P. Zanchetta, are with the PEMC group, University of Nottingham, Nottingham, NG7 2RD, UK (email: eexgv@exmail.nottingham.ac.uk). 
in Fig. 1 featuring a low coupling between winding sets. Table I reports the main characteristic of the motor.

Table I

MACHINE PARAMETERS

\begin{tabular}{ll}
\hline \multicolumn{1}{c}{ Parameter } & \multicolumn{1}{c}{ Value } \\
\hline Pole number $(2 p)$ & 6 \\
PM material & $\mathrm{NdFeB}$ \\
Magnet remanence $B_{r}$ & $1.15[\mathrm{~T}]$ \\
Magnet relative recoil permeability $\mu_{r}$ & 1.03 \\
Magnetization type & Radial \\
Turn/coil & 22 \\
Torque constant $\left(k_{T}\right)$ & $0.128[\mathrm{Nm} / \mathrm{A}]$ \\
Line to line voltage constant $\left(k_{V}\right)$ & $15.5\left[\mathrm{~V}_{\mathrm{pk}} / \mathrm{krpm}\right]$ \\
\hline
\end{tabular}

GEOMETRICAL PARAMETERS

\begin{tabular}{ll}
\hline \hline Axial length $l_{a}$ & $91[\mathrm{~mm}]$ \\
Inner Stator radius $R_{s}$ & $24.75[\mathrm{~mm}]$ \\
Mid-airgap Radius $R_{g}$ & $24.25[\mathrm{~mm}]$ \\
Air-gap length $g_{0}$ & $1[\mathrm{~mm}]$ \\
Magnets thickness $h_{m}$ & $4[\mathrm{~mm}]$ \\
Magnet pole-arc to pole-pitch ratio $\alpha_{p}$ & 0.78 \\
Slot-opening $b_{0}$ & $2.1[\mathrm{~mm}]$
\end{tabular}

\section{B. Mathematical model of MSPMS motor}

This section is a brief summary that provides the basis of the mathematical model presented in [11]. In this analysis the rotor is considered centered and supported by stiff mechanical bearings.

Under the assumptions of linear magnetic behavior of the materials and magnetic decoupling between sectors, the matrix formulation (1) expresses the generalized mechanical wrench of the motor [13] as a function of the stationary reference frame current components ${ }^{s} i_{\alpha}$ and ${ }^{s} i_{\beta}$ of each sector $s$.

$$
W_{E}\left(\theta_{e}\right)=\boldsymbol{K}_{E, 0}\left(\theta_{e},{ }^{s} \gamma\right) I_{\alpha \beta}
$$

where $W_{E}\left(\theta_{e}\right)=\left[\begin{array}{lll}F_{x}\left(\theta_{e}\right) & F_{y}\left(\theta_{e}\right) & T\left(\theta_{e}\right)\end{array}\right]^{T}$ is the vector collecting the mechanical forces and torque, while $I_{\alpha \beta}=$ $\left[\begin{array}{llllllll}{ }^{1} i_{\alpha} & { }^{1} i_{\beta} & \ldots & { }^{s} i_{\alpha} & { }^{s} i_{\beta} & \ldots & { }^{n} i_{\alpha} & { }^{n} i_{\beta}\end{array}\right]^{T}$ is the vector of the $\alpha-$ $\beta$ axis currents. ${ }^{s} \gamma$ is the geometrical angular position of the windings magnetic axis with respect the $\mathrm{x}$-axis and $\theta_{e}\left(\theta_{e}=\right.$ $\left.p \theta_{m}\right)$ is the electrical angular position of the rotor magnetic axis with respect the winding magnetic axis. The $3 \times 2 n_{s}$ $\boldsymbol{K}_{E, 0}$ matrix can be expressed in (2).

$$
\boldsymbol{K}_{E, 0}\left(\theta_{e},{ }^{s} \gamma\right)=\left[{ }^{1} \boldsymbol{K}_{E, 0}\left(\theta_{e},{ }^{1} \gamma\right) \cdots{ }^{n_{s}} \boldsymbol{K}_{E, 0}\left(\theta_{e},{ }^{n_{s}} \gamma\right)\right]
$$

where the $3 \times 2$ matrix ${ }^{s} \boldsymbol{K}_{E, 0}\left(\theta_{e},{ }^{s} \gamma\right)$ of the generic sector $s$ can be found in [11]. It consists of a $2 \times 2$ sub-matrix ${ }^{s} \boldsymbol{K}_{E, F, 0}\left(\theta_{e},{ }^{s} \gamma\right)$ of the force coefficients and of a $1 \times 2$ submatrix ${ }^{s} \boldsymbol{K}_{E, T}\left(\theta_{e}\right)$ of the torque coefficients. Each machine sector presents the same sub-matrix ${ }^{s} \boldsymbol{K}_{E, T}\left(\theta_{e}\right)$ since the torque coefficients only depend on the torque constant $k_{T}$ and rotor position $\theta_{e}$ [11]. On the other hand, sub-matrix ${ }^{s} \boldsymbol{K}_{E, F, 0}\left(\theta_{e},{ }^{s} \gamma\right)$ has to be determined for each machine sector. Levering on the geometrical and electromagnetic symmetries of the motor, ${ }^{s} \boldsymbol{K}_{E, F, 0}\left(\theta_{e},{ }^{s} \gamma\right)$ can be expressed with respect of the sub-matrix ${ }^{1} \boldsymbol{K}_{E, F, 0}\left(\theta_{e},{ }^{1} \gamma\right)$ of the reference sector (sector 1). As a matter of fact, each machine sector can be
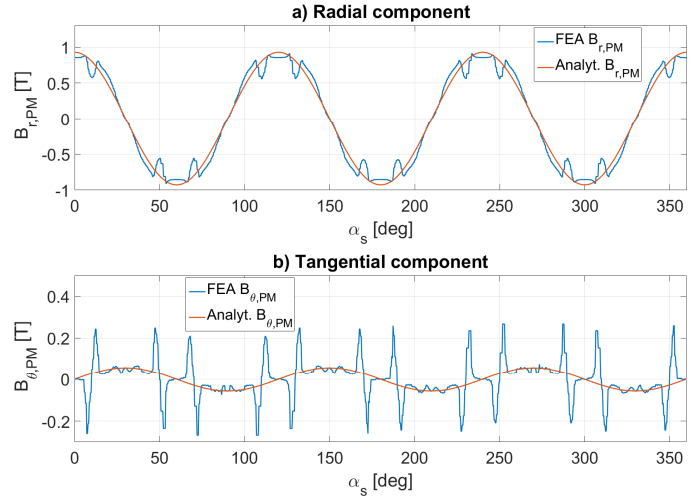

Fig. 2. Open-circuit airgap flux density for $\theta_{e}=0$ [deg].

expressed by multiplying ${ }^{1} \boldsymbol{K}_{E, F, 0}\left(\theta_{e},{ }^{1} \gamma\right)$ by an appropriate bi-dimensional rotation matrix $\boldsymbol{R}\left({ }^{s} \gamma\right)$ defined in [11].

The current commands required for the motor control can be evaluated inverting matrix $\boldsymbol{K}_{E, 0}$. However, $\boldsymbol{K}_{E, 0}$ do not result in a square matrix, hence in [11] a proper strategy has been proposed to optimize the current reference signals targeting the joule losses minimization. The Pseudo Inverse of $\boldsymbol{K}_{E, 0}$ has been introduced in [11] for this purpose and defined as in (3).

$$
K_{E, 0}^{+}=K_{E, 0}^{T}\left(K_{E, 0} K_{E, 0}^{T}\right)^{-1}
$$

The coefficients of the pseudo inverse matrix $\boldsymbol{K}_{\boldsymbol{E}, \mathbf{0}}^{+}$can be calculated offline to drastically reduce the online computation effort needed [11]. The optimized current reference signals can be therefore expressed as in (4).

$$
\bar{I}_{\alpha \beta}^{\prime}=\boldsymbol{K}_{E, 0}^{+}\left(\theta_{e}\right) \bar{W}_{E}^{*}
$$

In the following sections, a detailed analytical method for the calculation of the force coefficients of ${ }^{1} \boldsymbol{K}_{E, F}\left(\theta_{e},{ }^{1} \gamma\right)$ will be presented. In addition, the rotor displacement will be taken into account allowing to rewrite (1) in a more comprehensive formulation.

\section{FLUX DENSITY PREDICTION}

This section describes the analytical method used to predict the open-circuit and armature-reaction flux density at the airgap for both centered and eccentric rotor position. The analytical calculation of the coefficients of matrix ${ }^{1} \boldsymbol{K}_{E, F}\left(\theta_{e},{ }^{1} \gamma\right)$ can be carried out from the main airgap flux density and enables to develop the mathematical model considering the rotor eccentricity.

The model is generated assuming 2D flux density distribution. Under the assumption of linear magnetic behavior of the soft magnetic materials, the superimposition principle can be applied. Therefore, the flux density at the mid-airgap can be written in polar coordinates as in (5) featuring the dependency with respect of the rotor angular position $\theta_{e}$ and stator angular coordinate $\alpha_{s}$

$$
\begin{aligned}
& B_{r}\left(\alpha_{s}, \theta_{e}\right)=B_{r, P M}\left(\alpha_{s}, \theta_{e}\right)+B_{r, w}\left(\alpha_{s}, \theta_{e}\right) \\
& B_{\vartheta}\left(\alpha_{s}, \theta_{e}\right)=B_{\vartheta, P M}\left(\alpha_{s}, \theta_{e}\right)+B_{\vartheta, w}\left(\alpha_{s}, \theta_{e}\right)
\end{aligned}
$$

where $B_{r}$ and $B_{\vartheta}$ are the radial and tangential components of the flux density at the mid-airgap respectively. $B_{r, P M}$, $B_{\vartheta, P M}$ and $B_{r, w}, B_{\vartheta, w}$ are the radial and tangential components of the open-circuit and armature-reaction flux density at the mid-airgap, respectively. 

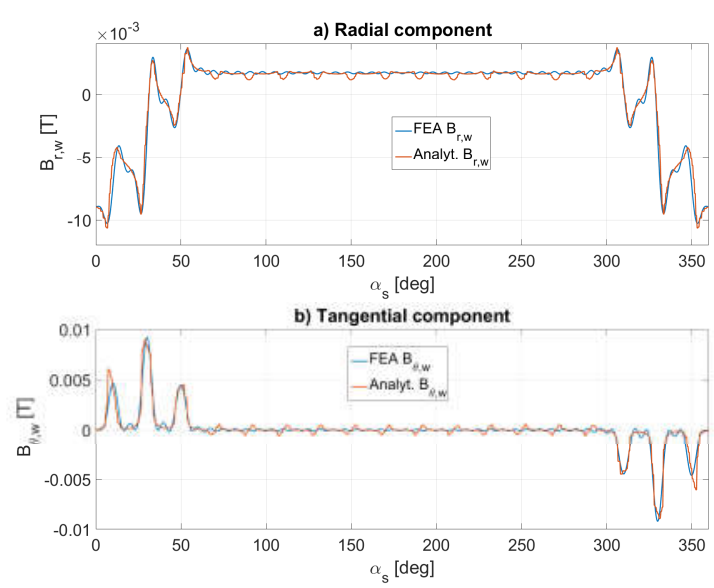

Fig. 3. -Armature-reaction flux density at the airgap produced by ${ }^{1} i_{\alpha}=$ const. $=1[A]$ and ${ }^{1} i_{\beta}=0[A]$.
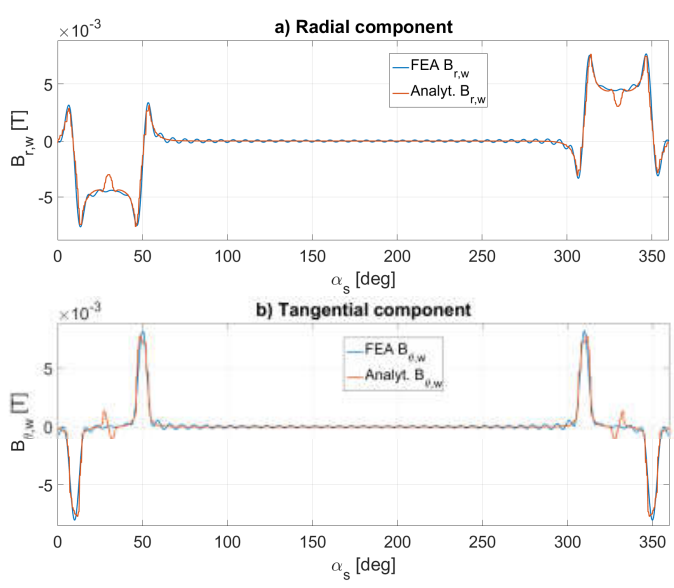

Fig. 4. -Armature-reaction flux density at the airgap produced by ${ }^{1} i_{\alpha}=$ $0[A]$ and ${ }^{1} i_{\beta}=$ const. $=1[A]$.

\section{A. No Rotor Displacement}

A comprehensive calculation of the open circuit flux density in the airgap for the case of perfectly centered rotor is carried out in [4]. The expression, in polar coordinate, is reported in (6).

$$
\begin{aligned}
& B_{r, P M}\left(\alpha_{s}, \theta_{e}\right)=\sum_{n=1,3,5 \ldots}^{\infty} K_{B}(n) f_{B r} \cos \left(n p\left(\alpha_{s}-\theta_{e}\right)\right) \\
& B_{\vartheta, P M}\left(\alpha_{s}, \theta_{e}\right)=\sum_{n=1,3,5 \ldots}^{\infty} K_{B}(n) f_{B \theta} \sin \left(n p\left(\alpha_{s}-\theta_{e}\right)\right)
\end{aligned}
$$

where $p$ is the number of pole pairs. Coefficients $K_{B}(n), f_{B r}$ and $f_{B \theta}$ take into account the main geometry features of the motor listed in Table I. Their expressions can be found in [12]. The flux density is calculated at the midairgap radius $R_{g}$. In Fig. 2 the flux density components obtained using equation (6) are compared with FEA for $\theta_{e}=$ 0 and $n=1$.

As far as the flux density due to the armature-reaction is concerned, this manuscript adapts the results of [5], achieved considering a conventional three-phase winding, to the multisector motor presented. Furthermore, only one machine sector (sector 1) has to be characterized in order to calculate the coefficients of the matrix $\boldsymbol{K}_{E, 0}\left(\theta_{e},{ }^{s} \gamma\right)$.



Fig. 5. Cross-section of the eccentric rotor with stator and rotor reference frames.

Equation (7) shows the radial and tangential components of the flux density produced by one motor phase.

$$
\begin{aligned}
& B_{r, p h}\left(\alpha_{s}, \theta_{e}\right)=K \sum_{v}^{\infty} \frac{1}{v} K_{s o v} K_{p v} F_{v}(r) I_{r}\left(\alpha_{s}, \theta_{e}\right) \\
& B_{\vartheta, p h}\left(\alpha_{s}, \theta_{e}\right)=K \sum_{v}^{\infty} \frac{1}{v} K_{s o v} K_{p v} G_{v}(r) I_{\vartheta}\left(\alpha_{s}, \theta_{e}\right)
\end{aligned}
$$

With $K=2 \frac{\mu_{0}}{\pi} \frac{N_{s}}{g_{t}}, I_{r}\left(\alpha_{s}, \theta_{e}\right)=i\left(\theta_{e}\right) \cos \left(v \alpha_{s}+\varphi_{p h}\right)$, $I_{\vartheta}\left(\alpha_{s}, \theta_{e}\right)=i_{p h}\left(\theta_{e}\right) \sin \left(v \alpha_{s}+\varphi_{p h}\right)$ and $v=1,2,3,4, \ldots \infty$. Coefficients $K_{s o v}, K_{p v}, F_{v}$ and $G_{v}$ can be found in [12], $i_{p h}(t)$ is the phase current, $g_{t}=g_{0}+h_{m}$ is the total airgap length (including the magnet thickness $h_{m}$ and the physical air-gap clearance $g_{0}$ ) and $\varphi_{p h}$ is the mechanical angular position of the phase axis with respect the $\mathrm{x}$-axis (for instance, for sector 1: $\left.\varphi_{u_{1}}=0, \varphi_{v_{1}}=-20[\mathrm{deg}], \varphi_{w_{1}}=20[\mathrm{deg}]\right)$.

The radial and tangential flux density distributions of one winding sector ( ${ }^{s} B_{r, w}$ and ${ }^{s} B_{\vartheta, w}$ ) can be obtained summing up the contributions of the phases given by (7) and updating $\varphi_{p h}$ according to the phase considered. However, only ${ }^{1} B_{r, w}$ and ${ }^{1} B_{\vartheta, w}$ are sufficient to express the sub-matrix ${ }^{1} \boldsymbol{K}_{E, F, 0}\left(\theta_{e},{ }^{1} \gamma\right)$ of the force coefficients. Fig. 3 and Fig. 4 show ${ }^{1} B_{r, w}$ and ${ }^{1} B_{\vartheta, w}$ obtained analytically (for $v=$ $1, \ldots, 50)$ and with FEA for ${ }^{1} i_{\alpha}=$ const. $=1[A],{ }^{1} i_{\beta}=0[A]$ and ${ }^{1} i_{\alpha}=0[A], \quad{ }^{1} i_{\beta}=$ const. $=1[A]$, respectively. The three-phase current pattern can be obtained applying the inverse Clarke's transformation [14]. Since the stator and the rotor are considered isotropic ${ }^{1} B_{r, w}$ and ${ }^{1} B_{\vartheta, w}$ are equal $\forall \theta_{e}$.

\section{B. Rotor Displacement}

The flux density prediction in the airgap region in the case of static rotor eccentricity is the topic of this section. Fig. 5 shows a generic rotor displacement geometrical description adopted in further calculations. The rotor axis $O_{r}$ appears to be translated of the quantity $\delta$ and angle $\varphi_{d}$ from the stator axis $O_{s}$.

In order to predict the flux density distribution in the airgap when rotor eccentricity occurs, the constant inner stator radius $R_{s}$ and middle airgap radius $R_{g}$ have to be replaced by variable distances $R_{s r}$ and $R_{g r}$, respectively. Furthermore $\alpha_{s}$ has to be 
updated with $\alpha_{s r}$ [8], [9]. This is the result of the coordinate transformation shown in Fig. 5 of a generic point $P$ on the stator surface or at the mid-airgap from the stator to the rotor reference frame.The aforementioned transformation consists of a rotation (8) and a translation (9).

$$
\begin{aligned}
& {\left[\begin{array}{l}
P^{\prime}{ }_{x r} \\
P^{\prime}{ }_{y r}
\end{array}\right]=\left[\begin{array}{cc}
\cos \left(\varphi_{d}\right) & \sin \left(\varphi_{d}\right) \\
-\sin \left(\varphi_{d}\right) & \cos \left(\varphi_{d}\right)
\end{array}\right]\left[\begin{array}{l}
P_{x s} \\
P_{y s}
\end{array}\right]} \\
& P_{x r}=P^{\prime}{ }_{x r}-\delta \quad P_{y r}=P^{\prime}{ }_{y r}
\end{aligned}
$$

Where $P_{x s}=R_{s} \cos \left(\alpha_{s}\right)$ and $P_{y s}=R_{s} \sin \left(\alpha_{s}\right)$ are the Cartesian coordinates of the point $P$ in the stator reference frame while $P_{x r}$ and $P_{y r}$ are the ones in the rotor reference frame. $R_{s r}$ and $\alpha_{s r}$ can be now calculated in (10).

$$
R_{s r}=\sqrt{{P_{x r}{ }^{2}+P_{y r}{ }^{2}}^{2}} \quad \alpha_{s r}=\tan ^{-1} \frac{P_{y r}}{P_{x r}}
$$

$R_{g r}$ can be obtained in the same way using (8), (9) and (10) replacing the inner stator radius $R_{S}$ with the mid-airgap radius $R_{g}$. From Fig. 5, the angle $\alpha_{s}$ can be written in (11) taking into account the transformation introduced.

$$
\alpha_{s} \approx \alpha_{s r}+\varphi_{d}
$$

Finally, the non-uniform airgap length can be found in (12) [9].

$$
g\left(\alpha_{s}\right)=g_{0}+e \cdot \cos \left(\alpha_{s}-\varphi_{d}\right)
$$

\section{FORCE COEFFICIENTS CALCULATION}

\section{A. Force coefficients for zero rotor displacement}

This section deals with the analytical calculation of the coefficients of matrix ${ }^{1} \boldsymbol{K}_{E, F, 0}\left(\theta_{e},{ }^{1} \gamma\right)$ presented in II. B.

The coefficients can be computed using the Maxwell stress tensor based on the flux density predicted in the previous sections. The radial and tangential stresses are given by (13) [10].

$$
\begin{gathered}
\sigma=\frac{B_{r}^{2}-B_{\vartheta}^{2}}{2 \mu_{0}} \\
\tau=\frac{B_{r} B_{\vartheta}}{\mu_{0}}
\end{gathered}
$$

where $B_{r}$ and $B_{\vartheta}$ can be calculated in (5) as the sum of the open-circuit and armature-reaction contributions.

Knowing the radial and tangential stress tensors from (13), the expressions of the coefficients of ${ }^{1} \boldsymbol{K}_{E, F, 0}\left(\theta_{e},{ }^{1} \gamma\right)$ are given by (14).

${ }^{1} k_{x, *, 0}=R_{g} l_{a}\left(\int_{0}^{2 \pi} \sigma \cos \left(\alpha_{s}\right) d \alpha-\int_{0}^{2 \pi} \tau \sin \left(\alpha_{s}\right) d \alpha_{s}\right)$

${ }^{1} k_{y, *, 0}=R_{g} l_{a}\left(\int_{0}^{2 \pi} \sigma \sin \left(\alpha_{s}\right) d \alpha+\int_{0}^{2 \pi} \tau \cos \left(\alpha_{s}\right) d \alpha_{s}\right)$

In (14), subscription * stands for either $\alpha$ - or $\beta$-axis current. As a matter of fact, four coefficients are required to define the $2 \times 2$ matrix ${ }^{1} \boldsymbol{K}_{E, F, 0}:{ }^{1} k_{x, \alpha, 0},{ }^{1} k_{y, \alpha, 0},{ }^{1} k_{x, \beta, 0}$ and ${ }^{1} k_{y, \beta, 0}$. Each of them can be obtained adopting a proper current pattern for the armature-flux density prediction in (7). For instance, ${ }^{1} k_{x, \alpha}$ and ${ }^{1} k_{y, \alpha}$ are obtained with ${ }^{1} i_{\alpha}=$ const. $=1[A]$, ${ }^{1} i_{\beta}=0[A]$ and ${ }^{1} k_{x, \beta}$ and ${ }^{1} k_{y, \beta}$ with ${ }^{1} i_{\alpha}=0[A]$ and ${ }^{1} i_{\beta}=$ const. $=1[A]$. In [11] the trends of the four coefficients is presented and compared with the ones obtained with the FEA showing good agreement in the results. Their sinusoidal approximated expressions are reported in (15).

$$
\left\{\begin{aligned}
{ }^{1} k_{x, \alpha, 0}\left(\theta_{e}, 0\right) & ={ }^{1} \tilde{k}_{x, \alpha, 0} \cos \left(\theta_{e}+\varphi_{x, \alpha}\right) \\
{ }^{1} k_{x, \beta, 0}\left(\theta_{e}, 0\right) & ={ }^{1} \tilde{k}_{x, \beta, 0} \cos \left(\theta_{e}+\varphi_{x, \beta}\right) \\
{ }^{1} k_{y, \alpha, 0}\left(\theta_{e}, 0\right) & ={ }^{1} \tilde{k}_{y, \alpha, 0} \cos \left(\theta_{e}+\varphi_{y, \alpha}\right) \\
{ }^{1} k_{y, \beta, 0}\left(\theta_{e}, 0\right) & ={ }^{1} \tilde{k}_{y, \beta, 0} \cos \left(\theta_{e}+\varphi_{y, \beta}\right)
\end{aligned}\right.
$$

where $\tilde{k}_{*, \times, 0}$ and $\varphi_{*, \times, 0}$ are the amplitude and phase shift of the force coefficients of the reference sector. Their numerical values can be found in [11] and are reported in Table II.

\section{Table II}

Force Coefficients Parameters

\begin{tabular}{c|cc|cc} 
& $x-\alpha$ & $x-\beta$ & $y-\alpha$ & $y-\beta$ \\
\hline \hline$\tilde{k}_{*, \times, 0}$ & 8.6 & 9.2 & 0.7 & 4.3 \\
$\varphi_{*, \times, 0}$ & $\pi$ & $\pi / 2$ & $-\pi / 2$ & $\pi$ \\
$\Delta_{*, \times}$ & 0.64 & 0.81 & 0.2 & 0.15
\end{tabular}

In the next section, ${ }^{1} k_{x, \alpha, 0},{ }^{1} k_{y, \alpha, 0},{ }^{1} k_{x, \beta, 0}$ and ${ }^{1} k_{y, \beta, 0}$ are calculated taking into account the rotor displacement. Hence, a new $2 \times 2$ matrix ${ }^{1} K_{E, F, \delta}$, function not only of $\theta_{e}$ and ${ }^{1} \gamma$ but also the dependency with respect $\delta$ and $\varphi_{d}$, will be introduced. Furthermore, the matrix formulation (1) will be modified introducing the stiffness matrix $G\left(\varphi_{d}\right)$ to include the rotor displacement.

\section{B. Force coefficients with rotor displacement}

A maximum displacement of $\delta_{\max }=0.25 \mathrm{~mm}(25 \%$ of the airgap $g_{0}$ ) has being chosen in order to fulfill the force capability of the motor. The amplitude of the UMP at $\delta_{\max }$ is around $165[\mathrm{~N}]$, corresponding to almost $90 \%$ of the force capability of the motor.

The force coefficients of the $2 \times 2$ matrix ${ }^{1} \boldsymbol{K}_{E, F, \delta}$ can still be calculated using (14) in the case of rotor displacement. However, the flux density required to calculate the radial and tangential stresses in (13) has to take into account the procedure of section III. B. Furthermore, the open-circuit X$\mathrm{y}$ force components have to be calculated in advanced so that the force coefficients can be obtained by (16).

$$
\begin{aligned}
{ }^{1} k_{x, *, \delta} & =\frac{\left(F_{x, *}-F_{x, o c}\right)}{{ }^{1} i_{*}} \\
{ }^{1} k_{y, *, \delta} & =\frac{\left(F_{y, *}-F_{y, o c}\right)}{{ }^{1} i_{*}}
\end{aligned}
$$

where the subscript $*$ stands for either $\alpha$ - or $\beta$-axis current. $F_{x, o c}$ and $F_{y, o c}$ are the open-circuit $\mathrm{x}-\mathrm{y}$ force components obtained with (14) taking into account the rotor displacement and imposing the armature-reaction flux density components $B_{r, w}$ and $B_{\vartheta, w}$ equal to zero. The analytical and FE trends of $F_{x, o c}$ and $F_{y, o c}$ versus $\delta$ and $\varphi_{d}$ are shown in Fig. 6. It allows 


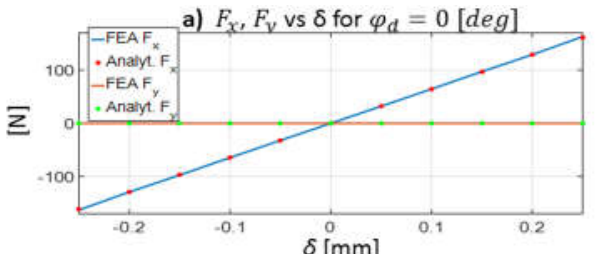

b) $F_{x}, F_{y}$ vs $\delta$ for $\varphi_{d}=30[\mathrm{deg}]$
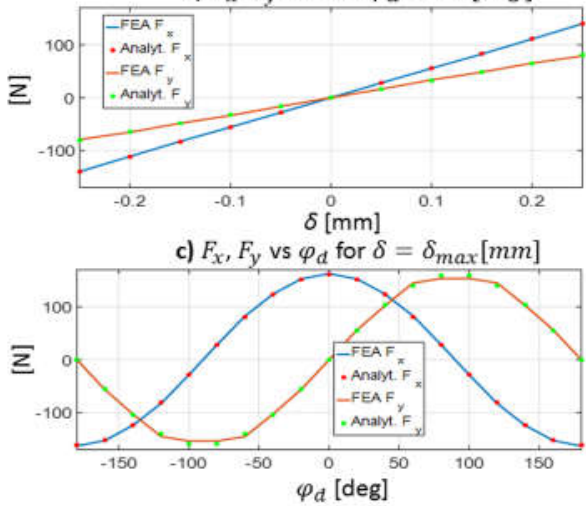

Fig. 6 Open-circuit $\mathrm{x}-\mathrm{y}$ force components.

defining the stiffness matrix $G\left(\varphi_{d}\right)$ as follow:

$$
G\left(\varphi_{d}\right)=\left[\begin{array}{l}
k \cos \left(\varphi_{d}\right) \\
k \sin \left(\varphi_{d}\right)
\end{array}\right]
$$

where $k=655 \mathrm{kN} / \mathrm{m}$.

Coefficients ${ }^{1} k_{x, \alpha, \delta}, \quad{ }^{1} k_{y, \alpha, \delta},{ }^{1} k_{x, \beta, \delta}$ and $\quad{ }^{1} k_{y, \beta, \delta}$ result function not only of the angular rotor position $\theta_{e}$ but also of the displacement information $\delta$ and $\varphi_{d}$. Fig. 8 shows the coefficient amplitudes as a function of $\delta$ and $\varphi_{d}$ analytically calculated considering the already mentioned $\alpha-\beta$ current supplies. The compact and approximated expressions of the force coefficients amplitudes for the generic sector $s$ is given in (18) including the information of the angular position ${ }^{s} \gamma$ of the sector $s$ winding.

$$
\begin{aligned}
& { }^{s} \tilde{k}_{x, *, \delta}\left(\delta, \varphi_{d},{ }^{s} \gamma\right)={ }^{s} \tilde{k}_{x, *, 0}+\Delta_{x, *} \frac{\delta}{\delta_{\max }} \cos \left(\varphi_{d}+{ }^{s} \gamma\right) \\
& { }^{s} \tilde{k}_{y, *, \delta}\left(\delta, \varphi_{d},{ }^{s} \gamma\right)={ }^{s} \tilde{k}_{y, *, 0}+\Delta_{y, *} \frac{\delta}{\delta_{\max }} \cos \left(\varphi_{d}+{ }^{s} \gamma\right)
\end{aligned}
$$

Where the amplitudes ${ }^{s} \tilde{k}_{x, *, 0}$ and ${ }^{s} \tilde{k}_{y, *, 0}$ for centered rotor can be obtained multiplying the force matrix ${ }^{1} \boldsymbol{K}_{E, F, 0}$ of the reference sector by the rotation matrix $\boldsymbol{R}\left({ }^{s} \gamma\right) . \Delta_{x, *}=$ ${ }^{s} \tilde{k}_{x, *, \max }-{ }^{s} \tilde{k}_{x, *, 0}$ and $\Delta_{y, *}={ }^{s} \tilde{k}_{y, *, \max }-{ }^{s} \tilde{k}_{y, *, 0}$. The numerical values of the above introduced coefficients are given in Table II. It is straightforward to notice from Table II that the main contribution to the force production is given by coefficients ${ }^{1} k_{x, \alpha, 0}$ and ${ }^{1} k_{x, \beta, 0}$. Furthermore, it is possible to conclude from the same table that the percentages of increment $\left(\Delta_{x, *} /{ }^{1} k_{x, *, 0}\right)$ of their amplitudes at $\delta_{\max }$ are relatively small. Their values are around $7.5 \%$ and $8.8 \%$, respectively. Therefore, the matrix of the force coefficients ${ }^{s} \boldsymbol{K}_{E, F, \delta}\left(\theta_{e},{ }^{s} \gamma, \delta, \varphi_{d}\right)$ can be approximated by the matrix ${ }^{1} \boldsymbol{K}_{E, F, 0}\left(\theta_{e},{ }^{1} \gamma\right)$ calculated with a centered rotor. A further motivation for this approximation can be found in the fact that the resulting small average error in the force production can be easily compensated by a standard PI controller in the case a)

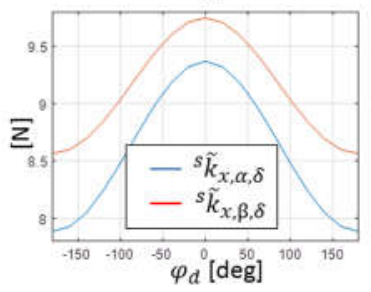

c)
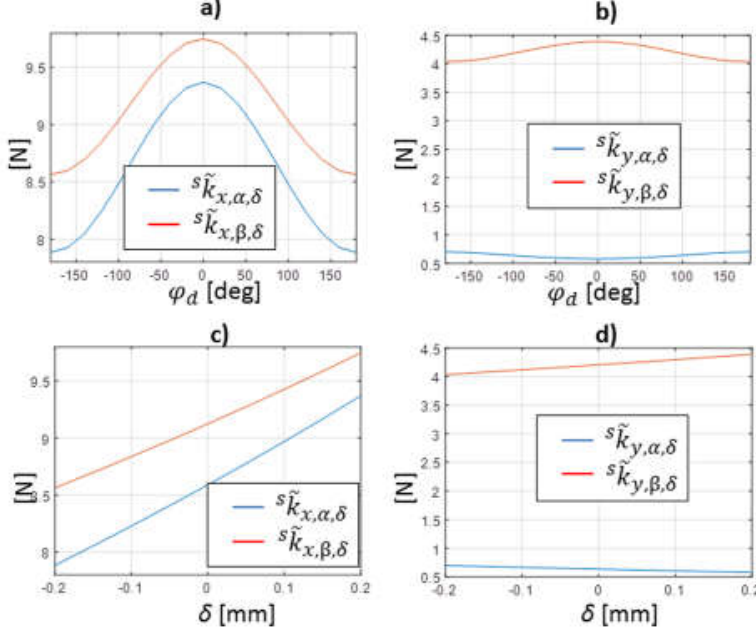

d)

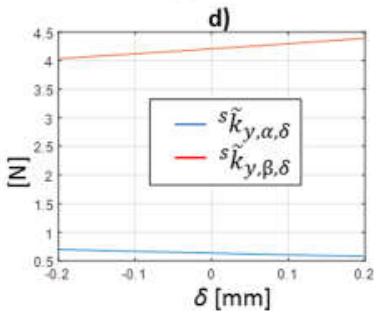

Fig. 8. Amplitude of the force coefficients of the reference sector.

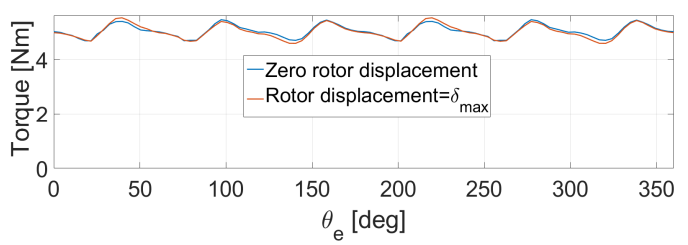

Fig. 7. Torque production for centered and eccentric rotor $\left(\delta=\delta_{\max }\right)$.

of static eccentricity. Additionally, the pseudo inverse of $\boldsymbol{K}_{E, 0}$ can be still calculated offline using (3) reducing the computational efforts of the control platform.

\section{Mathematical model including rotor eccentricity}

This section presents a new mathematical expression that takes into account the rotor displacement. In (1) a simple matrix formulation of the mechanical outputs as a function of the $\alpha-\beta$ currents was given assuming a centered rotor. In order to include an eventual rotor displacement, the stiffness term has to be added in the formulation to account for the no load $x-y$ force components. Thanks to the approximation introduced in the previous section, the rotor displacement effect can be neglected in the force coefficients. Furthermore, Fig. 7 shows the FE simulation result for nominal torque production $\left(T_{\text {nominal }}=5 \mathrm{Nm}\right)$ for both zero and maximum rotor displacement. It can be observed that the torque production is almost the same, only a small difference in the torque ripple can be appreciated, justifying the further approximation of considering the sub-matrix ${ }^{s} \boldsymbol{K}_{E, T}$ independent from the rotor displacement. Hence, matrix $\boldsymbol{K}_{E, 0}$, defined in (2), can still be used provided that $\delta \leq \delta_{\max }$. The new matrix formulation of the mathematical model is given in (19).

$$
W_{E}\left(\theta_{e}, \delta, \varphi_{d}\right)=\boldsymbol{K}_{E, 0}\left(\theta_{e}\right) I_{\alpha \beta}+G_{t}\left(\varphi_{d}\right) \delta
$$

Where $G_{t}\left(\varphi_{d}\right)$ is a three elements vector consisting in equation (17) for the first two elements and zero for the third. The vector of the currents required to develop the reference mechanical outputs with an eventual rotor displacement is now given by (20).

$$
\bar{I}_{\alpha \beta}^{\prime}=\boldsymbol{K}_{E, 0}^{+}\left(\bar{W}_{E}^{*}-G_{t}\left(\varphi_{d}\right) \delta\right)
$$



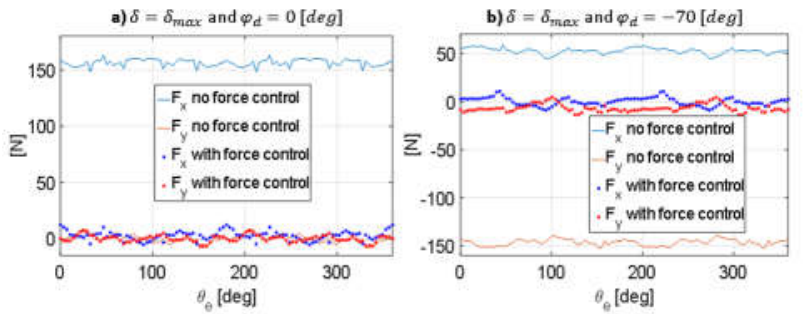

Fig. 9. $x-y$ force components generated with and without force control.

\section{Simulation Results}

In this section the simulation results carried out through FEA will be presented. The $\alpha-\beta$ current commands are obtained with (20) for the desired mechanical vector $\bar{W}_{E}^{*}$ and considering a certain rotor displacement. The $2 n_{s}$ phase currents result in applying the inverse Clarke transformation to vector $\bar{I}_{\alpha \beta}^{\prime}$ and can be used to supply the FE model of the motor. In the following simulations the rotor is shifted of the desired displacement from the centered position and rotated around its axis. A multi-static 2D non-linear simulation is adopted in order to take the motor core saturation into account. Fig. 9 shows the resultant force with and without the force control for 2 eccentricity conditions. It is possible to appreciate how the force control drastically reduces the UMP caused by the rotor displacement.

\section{CONCLUSION}

This paper presents a mathematical model that defines the mechanical output of the MSPMS motor considered in the case of rotor eccentricity. The mathematical model has been generated by analytically predicting the flux density at the mid-airgap for both centered and eccentric rotor and computing the result with the Maxwell stress tensor method. Finally, the mathematical model has been adopted to create a force and torque control technique that allows compensating the UMP caused by the rotor displacement. The results obtained applying the force and torque control technique shows an effective compensation of the UMP.

\section{REFERENCES}

[1] L. Papini, T. Raminosoa, D. Gerada, and C. Gerada, "A high-speed permanent-magnet machine for fault-tolerant drivetrains," IEEE Trans. Ind. Electron., vol. 61, no. 6, pp. 3071-3080, 2014.

[2] A. Formentini, L. D. Lillo, M. Marchesoni, A. Trentin, P. Wheeler, and P. Zanchetta, "A new mains voltage observer for PMSM drives fed by matrix converters," in 2014 16th Europ. Conf. on Power Electr. and Applic., 2014, pp. 1-10.

[3] D. Torregrossa, A. Khoobroo, and B. Fahimi, "Prediction of Acoustic Noise and Torque Pulsation in PM Synchronous Machines With Static Eccentricity and Partial Demagnetization Using Field Reconstruction Method," IEEE Transactions on Industrial Electronics, vol. 59, no. 2, pp. 934-944, 2012.

[4] Z. Q. Zhu, D. Howe, E. Bolte, and B. Ackermann, "Instantaneous magnetic field distribution in brushless permanent magnet DC motors. I. Open-circuit field," IEEE Trans. on Magnetics, vol. 29, no. 1, pp. 124-135, 1993.

[5] Z. Zhu, and D. Howe, "Instantaneous magnetic field distribution in brushless permanent magnet DC motors. II. Armature-reaction field," IEEE Trans. on Magn., vol. 29, no. 1, pp. 136-142, 1993.

[6] H. Mahmoud, and N. Bianchi, "Eccentricity in Synchronous Reluctance Motors-Part I: Analytical and Finite-Element Models," IEEE Trans. on Energy Conv., vol. 30, no. 2, pp. 745-753, 2015.
[7] H. Mahmoud, and N. Bianchi, "Eccentricity in Synchronous Reluctance Motors-Part II: Different Rotor Geometry and Stator Windings," IEEE Trans. on Energy Conv., vol. 30, no. 2, pp. 754-760, 2015.

[8] M. Michon, R. C. Holehouse, K. Atallah, and J. Wang, "Unbalanced magnetic pull in permanent magnet machines," in 7th IET Internat. Conf. on Power Electr.,Mach. and Drives (PEMD 2014),2014,pp. 1-6.

[9] H. Mahmoud, and N. Bianchi, "Analytical comparison of synchronous reluctance and surface permanent magnet machines with rotor eccentricity," in 2015 IEEE Energy Conversion Congress and Exposition (ECCE), 2015, pp. 1765-1772.

[10] Z. Q. Zhu, M. Mohd Jamil, and L. Wu, "Influence of slot and pole number combinations on unbalanced magnetic force in PM machines with diametrically asymmetric windings," IEEE Trans. on Ind. Applications, vol. 49, no. 1, pp. 19-30, 2013.

[11] G. Valente, L. Papini, A. Formentini, C. Gerada, and P. Zanchetta, "Radial force control of multi-sector permanent magnet machines," in 2016 XXII Intern. Conf. on Electr. Mach.(ICEM), 2016, pp. 2595-2601.

[12] Z. Zhu, Z. Xia, L. Wu, and G. Jewell, "Analytical modeling and finiteelement computation of radial vibration force in fractional-slot permanent-magnet brushless machines," IEEE Trans. on Industry Applications, vol. 46, no. 5, pp. 1908-1918, 2010.

[13] P. Bolognesi, "A mid-complexity analysis of long-drum-type electric machines suitable for circuital modeling," in Electrical Machines, 2008. ICEM 2008. 18th Intern. Conf. on,2008, pp. 1-5.

[14] E. Levi, R. Bojoi, F. Profumo, H. A. Toliyat, and S. Williamson, "Multiphase induction motor drives - a technology status review," IET Electric Power Applications, vol. 1, no. 4, pp. 489-516, 2007.

\section{BIOGRAPHIES}

Giorgio Valente received his Bachelor degree in Energy Engineering in 2011 and his Master degree in Electrical Engineering in 2014 both from the University of Padova, Italy. Between 2013 and 2014 he developed sensorless control techniques for PMSM for his Master thesis at the University of Oviedo, Spain. He is now working towards its Ph.D. with the Power Electronics, Machines and Control Group, University of Nottingham, UK. His main research interest is control of electrical machines.

Luca Papini received his Bachelor degree (Hons.) and Master degree (Hons.) in Electrical engineering in 2009 and 2011, respectively, both from the University of Pisa, Italy. He's been visiting student at The University of Nottingham, UK, developing analytical and numerical models for his Master thesis. From June to November 2011 he collaborated with the Department of Energy Engineering, University of Pisa, as a research assistant. He is currently working towards its Ph.D. with the Power Electronic, Motors and Drives Group at University of Nottingham. Since 2013 hold a position of research assistant in the same institution. His main research interests are high speed, high power density electric machines, machine control and levitating system.

Andrea Formentini was born in Genova, Italy, in 1985. He received the M.S. degree in computer engineering and the $\mathrm{PhD}$ degree in electrical engineering from the University of Genova, Genova, in 2010 and 2014 respectively. He is currently working as research fellow in the Power Electronics, Machines and Control Group, University of Nottingham. His research interests include control systems applied to electrical machine drives and power converters.

Chris Gerada (M'05) received the Ph.D. degree in numerical modeling of electrical machines from the University of Nottingham, Nottingham, U.K., in 2005. He subsequently worked as a Researcher at the University of Nottingham on high-performance electrical drives and on the design and modeling of electromagnetic actuators for aerospace applications. He was appointed as Lecturer in electrical machines in 2008, Associate Professor in 2011, and Professor in 2013. His core research interests include the design and modeling of high-performance electric drives and machines. Prof. Gerada is an Associate Editor of the IEEE Transaction on Industry Applications.

Pericle Zanchetta (M'00-SM'15) received his degree in Electronic Engineering and his Ph.D. in Electrical Engineering from the Technical University of Bari (Italy) in 1993 and 1997 respectively. In 1998 he became Assistant Professor of Power Electronics at the same University. In 2001 he became lecturer in control of power electronics systems in the PEMC research group at the University of Nottingham - UK, where he is now Professor in Control of Power Electronics systems. He has published over 200 peer reviewed papers and he is Vice-Chair of the IAS Industrial Power Converter Committee IPCC. His research interests include control of power converters and drives, Matrix and multilevel converters. 